\title{
Introduction to the Special Issue on AI and Networks
}

\author{
Marie desJardins, Matthew E. Gaston, \\ and Dragomir Radev
}

- This introduction to AI Magazine's special issue on networks and AI summarizes the seven articles in the special issue by characterizing the nature of the networks that are the focus of each of the articles. A short tutorial on graph theory and network structures is included for those less familiar with the topic.
Most people ... would agree that a fundamental property of complex systems is that they are composed of a large number of components or "agents," interacting in some way such that their collective behavior is not a simple combination of their individual behaviors.

- Mark Newman

$\mathrm{T}$ The importance of networks permeates the world today. From biology to social systems, from the brain to the Internet, networks play an important and central role in the way the world works. In the last 10 years, due in part to large increases in computational power, large-scale, real-world networks have received much attention from a variety of fields of study.

Within the artificial intelligence community, networks appear in some form in nearly every subdiscipline: knowledge representation, inference, learning, natural language processing, multiagent systems, analogical reasoning, and many others. The goals of this special issue are to provide a sampling of research efforts focused on how networks can be used in AI systems and to facilitate cross-communication among subdisciplines that are studying networks from different perspectives.

The seven papers we include here cover a broad range of network-inspired AI research-in natural language processing, data mining, the semantic web, peer-topeer networks, multiagent systems, analog networks, and the modern social network of the "blogosphere." Each article represents a snapshot of the area it describes; for example, the collective classification problem surveyed by Prithviraj Sen, Galileo Namata, Mustafa Bilgic, Lise Getoor, Brian Gallagher, and Tina EliassiRad is just one of many problems within the emerging research area of link mining. Moreover, networks are influential in many other areas of AI that are not represented here, including Bayesian networks and graphical models, sensor networks, swarm systems and cellular automata, graphical games, trust and reputation systems, and computational organizational design.

Table 1 summarizes the articles in this collection by characterizing the nature of the networks that are the focus of each of the seven papers.

\section{Basic Graph Theory}

In reading the articles presented here, some basics of graph and network theory may be useful for the reader who is not familiar with these terms. We start with some basic terminology.

A graph $G$ is defined to be a pair $(V, E)$, where $V$ is a vertex set and $E$ is an edge set (see following). The terms graph and network are often used interchangeably.

The finite vertex set $V$ is a set of descriptors for the vertices in the graph. Each vertex may just have an identifier, or it may have an arbitrarily complex set of attributes. The terms vertex and node are often used interchangeably. Depending on the 


\begin{tabular}{|c|c|c|c|c|}
\hline Authors & Topic & Nodes & Edges & Tasks \\
\hline $\begin{array}{l}\text { Radev and } \\
\text { Mihalcea }\end{array}$ & $\begin{array}{l}\text { Natural language } \\
\text { processing }\end{array}$ & $\begin{array}{l}\text { Words, word } \\
\text { senses, } \\
\text { sentences, } \\
\text { documents }\end{array}$ & $\begin{array}{l}\text { Cooccurrences, } \\
\text { collocations, syntactic } \\
\text { structure, lexical } \\
\text { similarity }\end{array}$ & $\begin{array}{l}\text { Analyze syntax, identify } \\
\text { lexical semantics, retrieve } \\
\text { and summarize text, extract } \\
\text { keywords }\end{array}$ \\
\hline $\begin{array}{l}\text { Berners-Lee and } \\
\text { Kagal }\end{array}$ & Semantic web & $\begin{array}{l}\text { Agents, terms, } \\
\text { ontologies }\end{array}$ & $\begin{array}{l}\text { Connections between } \\
\text { communities, subtask } \\
\text { relationships, ontological } \\
\text { relationships }\end{array}$ & $\begin{array}{l}\text { Disseminate knowledge, } \\
\text { construct and share } \\
\text { ontologies, provide and } \\
\text { request services, create new } \\
\text { communities }\end{array}$ \\
\hline $\begin{array}{l}\text { Menczer, Wu, } \\
\text { and Akavipat }\end{array}$ & $\begin{array}{l}\text { Peer-to-peer } \\
\text { networks }\end{array}$ & Agents & $\begin{array}{l}\text { "Social" connections } \\
\text { along which queries flow }\end{array}$ & $\begin{array}{l}\text { Locate relevant knowledge } \\
\text { sources, learn which peers } \\
\text { can answer queries }\end{array}$ \\
\hline $\begin{array}{l}\text { Pearce, Tambe, } \\
\text { and } \\
\text { Maheswaran }\end{array}$ & $\begin{array}{l}\text { Cooperative } \\
\text { multiagent } \\
\text { systems }\end{array}$ & Agents & $\begin{array}{l}\text { Interactions, joint reward } \\
\text { structures }\end{array}$ & $\begin{array}{l}\text { Multiagent plan } \\
\text { coordination, meeting } \\
\text { scheduling, teamwork (such } \\
\text { as RoboCup soccer) }\end{array}$ \\
\hline $\begin{array}{l}\text { Mattiussi, } \\
\text { Marbach, Dürr, } \\
\text { and Floreano }\end{array}$ & Analog networks & $\begin{array}{l}\text { Dynamic } \\
\text { devices }\end{array}$ & $\begin{array}{l}\text { Signal flows with varying } \\
\text { strength }\end{array}$ & $\begin{array}{l}\text { Synthesize and reverse- } \\
\text { engineer analog networks } \\
\text { (for example, gene } \\
\text { regulatory networks and } \\
\text { analog electronic circuits) }\end{array}$ \\
\hline $\begin{array}{l}\text { Finin, Joshi, } \\
\text { Kolari, Java, } \\
\text { Kale, and } \\
\text { Karandikar }\end{array}$ & Blogosphere & $\begin{array}{l}\text { Web pages, } \\
\text { blog postings, } \\
\text { bloggers, blog } \\
\text { sites }\end{array}$ & $\begin{array}{l}\text { Social networks, } \\
\text { comments, trackbacks; } \\
\text { advertisements, tags, RDF } \\
\text { data, metadata }\end{array}$ & $\begin{array}{l}\text { Recognize spam blogs } \\
\text { (splogs), find opinions on } \\
\text { topics, identify } \\
\text { communities of interest, } \\
\text { derive trust relationships, } \\
\text { detect influential bloggers }\end{array}$ \\
\hline $\begin{array}{l}\text { Sen, Namata, } \\
\text { Bilgic, Getoor, } \\
\text { Gallagher, and } \\
\text { Eliassi-Rad }\end{array}$ & $\begin{array}{l}\text { Social and } \\
\text { natural networks }\end{array}$ & $\begin{array}{l}\text { Entities (such } \\
\text { as scientific } \\
\text { articles) }\end{array}$ & $\begin{array}{l}\text { Relationships among the } \\
\text { entities (for example, } \\
\text { citations or cocitations) }\end{array}$ & $\begin{array}{l}\text { Perform collective } \\
\text { classification, construct } \\
\text { features for relational } \\
\text { classification }\end{array}$ \\
\hline
\end{tabular}

Table 1. An Overview of the Articles in This Special Issue.

application, nodes may also be referred to as agents or entities.

The finite edge set $E$ specifies the relationships between the vertices in the graph. Each edge $e \in E$ is a pair of vertices, which are called the end points of the edge. Edges may be ordered or unordered and also weighted or unweighted. A hyperedge may connect more than two vertices. Edges are often used to represent relations.

The degree of a node, $k_{i}$, is the number of edges that are connected to node $i$. In directed graphs, degree can be broken down into "in-degree" (number of edges coming into the node) and "out-degree" (number of edges pointing out of the node).

\section{Network Properties}

A number of properties prove to be useful in graph theory and social network theory for analyzing and understanding the behavior of graph structures.

The path length between two nodes is the minimum number of edges that must be traversed to move from one node to the other in the graph. The average path length is an average across all pairs of nodes in the graph.

Real-world graphs often exhibit short average path lengths, meaning that the average path length is less than would be expected in a random graph. This "small-world effect" was first recognized by Stanley Milgram (1967) in analyzing the number of hops it took for human subjects to send a piece of postal mail to a predefined destination by following only links to people whom they knew on a first name basis. This phenomenon is sometimes called "six degrees of separation," based on the hypothesis that any two people in the world can be connected by at most a six-link "chain" of acquaintances. A game created in the mid-1990s 
called Six Degrees of Kevin Bacon (find a short path connecting any given movie actor or actress to Kevin Bacon) in fact initiated some of the research work that led to the current boom in interest in network studies.

Several other properties are related to path length: The betweenness of a node $i$ is the number of other pairs of nodes $(j, k)$ whose shortest paths pass through $i$. The closeness of a node is the average shortest path to all other nodes in the graph. The diameter of a graph is the length of the longest of all shortest paths (that is, it is the maximal distance between any pair of nodes $(i, j)$ ).

Clustering measures are used to characterize the frequency of transitive relationships in networks (Newman 2003, Albert and Barabási 2002, Watts and Strogatz 1998). The clustering coefficient of a network is the ratio of triangles in a network (sets of three nodes that are all connected to each other) to the number of connected triples (sets of three nodes in which at least one node is connected to the other two).

Real-world networks often exhibit excess clustering, in the sense that they have a much higher (often two orders of magnitude or more) clustering coefficient than would be expected in a random graph of the same size (Newman 2003). This is because in many processes that generate networks, two nodes that are connected to a common neighbor are more likely to become connected.

The degree of a node is also sometimes called its degree centrality, since the number of edges that are connected to a node give an indication of how "central" it is to the network. The degree distribution of a network is the frequency of occurrence of nodes with each degree. A useful summary property is the network's average degree, which can be thought of as the density of the network. The normalized standard deviation of the degrees of the nodes can be used to characterize how much variability there is in the network density. The degree correlation of adjacent nodes in a network indicates whether neighboring nodes are likely to have similar degree.

Many real-world networks have highly skewed degree distributions, with high normalized standard deviation. In particular, the degree distribution in real-world networks often follows a power law, where the probability of a node in the network having degree $k$ is proportional to $k^{-\gamma}$ for some parameter $\gamma$ (typically $\gamma$ is between -2 and -3 ) (Newman 2003). Such networks have a hub-and-spoke structure, with some nodes having very large degree (Albert and Barabási 2002).

\section{Network Models}

A variety of network models have been proposed to represent various types of network formation processes and graph behaviors. Several of the most common models are described in the following paragraphs.

Regular graphs have a homogeneous connectivity pattern for all of the nodes in the graph. In these graphs, the degree distribution is trivial: all nodes have the same degree. Examples of regular graphs include lattices, hypercubes, and fully connected networks (in which all nodes are connected to all other nodes).

The coordination number (Watts 1999) of a lattice graph determines the number of connections that each node has with its spatial "nearest neighbors" in each dimension. An example of a one-dimensional lattice with $K=2$ is shown in figure 1(a).

Random graphs were first introduced by Paul Erdös and Alfréd Rényi (1959). A random graph $G_{n, p}$ consists of $n$ nodes where $p$ denotes the probability of an edge existing between each pair of vertices. Random graph models have been widely studied, in part because their properties can be computed analytically. For instance, the expected number of undirected edges in $G_{n, p}$ is $n(n-1) p / 2$, and the average degree of a vertex is $k=p(n-1)$.

A random geometric graph is a special case of a random graph that is generated by randomly placing $N$ nodes in the unit square, then connecting pairs of nodes if they are within some specified distance of each other (Dall and Christensen 2002). More specifically, two nodes, $i$ and $j$, are connected in a random geometric graph if $d(i, j)<\phi$, where $\phi$ is a threshold parameter of the model. Figure 2 shows an instance of a random geometric graph with $\phi=$ 0.09 .

The small-world network model of Duncan Watts and Steven Strogatz (1998) is an attempt to produce networks that exhibit the real-world properties of excess clustering and short average path length. Small-world networks have properties that lie between those of regular (lattice) networks and random graphs. Small-world networks are constructed by randomly "rewiring" each edge in a lattice network with some probability $\rho$. This process results in shortcut connections across the network, as seen in figure 1. (When edges are replaced with random shortcuts with probability $\rho=1$, the resulting graph is a random graph.)

The scale-free graph model is motivated by the empirically measured degree distributions of the Internet and the World Wide Web (Albert and Barabási 2002; Albert, Jeong, and Barabási 1999). The model is a highly intuitive model based on the way that many networks are believed to evolve and grow in the real world. The generation of scale-free graphs has two simple rules: (1) growth-at each time step, a new node is added to the graph, and (2) preferential attachment-when a new node is added to the graph, it attaches preferentially to existing nodes with high degree. Figure 3 shows an 


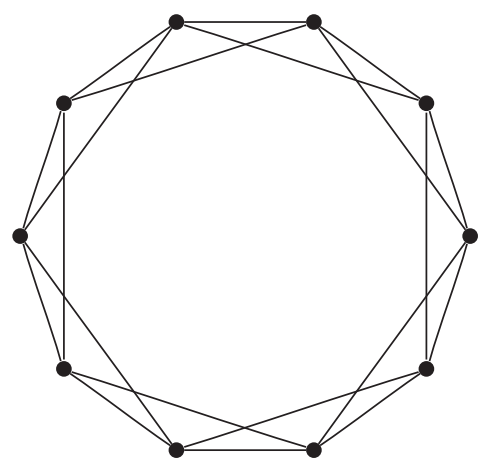

$\rho=0.0$

a

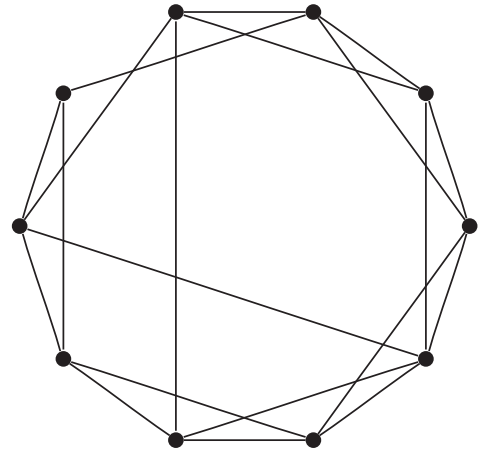

$\rho=0.1$

b

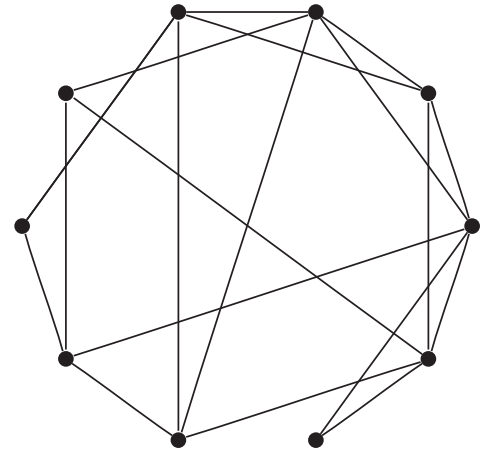

$\rho=0.3$

C

Figure 1. Three Increasingly Random Small-World Networks.

(a) A small world with no shortcut links. (b) The same small world with a few shortcuts. (c) A small world with many shortcuts, which begins to resemble a random graph. All three of the networks are constructed from a one-dimensional lattice where nodes are connected to $\mathrm{K}=2$ other nodes in each direction, based on physical proximity. This particular choice of initial layout is deliberate in that it ensures a high initial clustering coefficient.

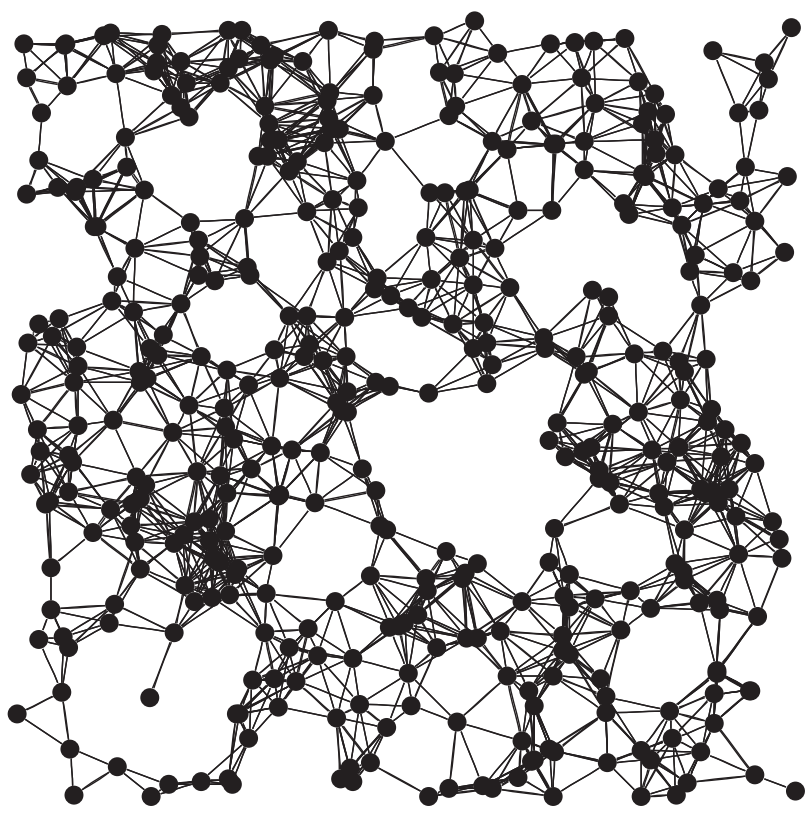

Figure 2. An Instance of a Random Geometric Graph on the Unit Square with 400 Nodes and $\phi=0.09$. example of a scale-free network structure and the power-law degree distribution that it exhibits.

\section{Summary}

Networks have been studied in artificial intelligence in a variety of contexts since AI's inception over 50 years ago. However, new insights from the study of very large real-world networks and from the connective power of the Internet call for an updated perspective on the importance and role of networks in AI. This special issue provides a sampling of the most innovative recent research in networks and AI.

\section{References}

Albert, R., and Barabási, A.-L. 2002. Statistical Mechanics of Complex Networks. Review of Modern Physics 99(3) (May): 7314-7316.

Albert, R.; Jeong, H.; and Barabási, A.-L. 1999. The Diameter of the World-Wide Web. Nature 401: 130-131, (10.1038/43601).

Dall, J., and Christensen, M. 2002. Random Geometric Graphs. Physical Review E, 66(016121): 1-9, July. (10.1103/PhysRevE.66.016121).

Erdös, P., and Rényi, A. 1959. On Random Graphs. Publicationes Mathematicae 6(Debrecen): 290-297.

Milgram, S. 1967. The Small World Problem. Psychology Today 22 (May): 61-67. 


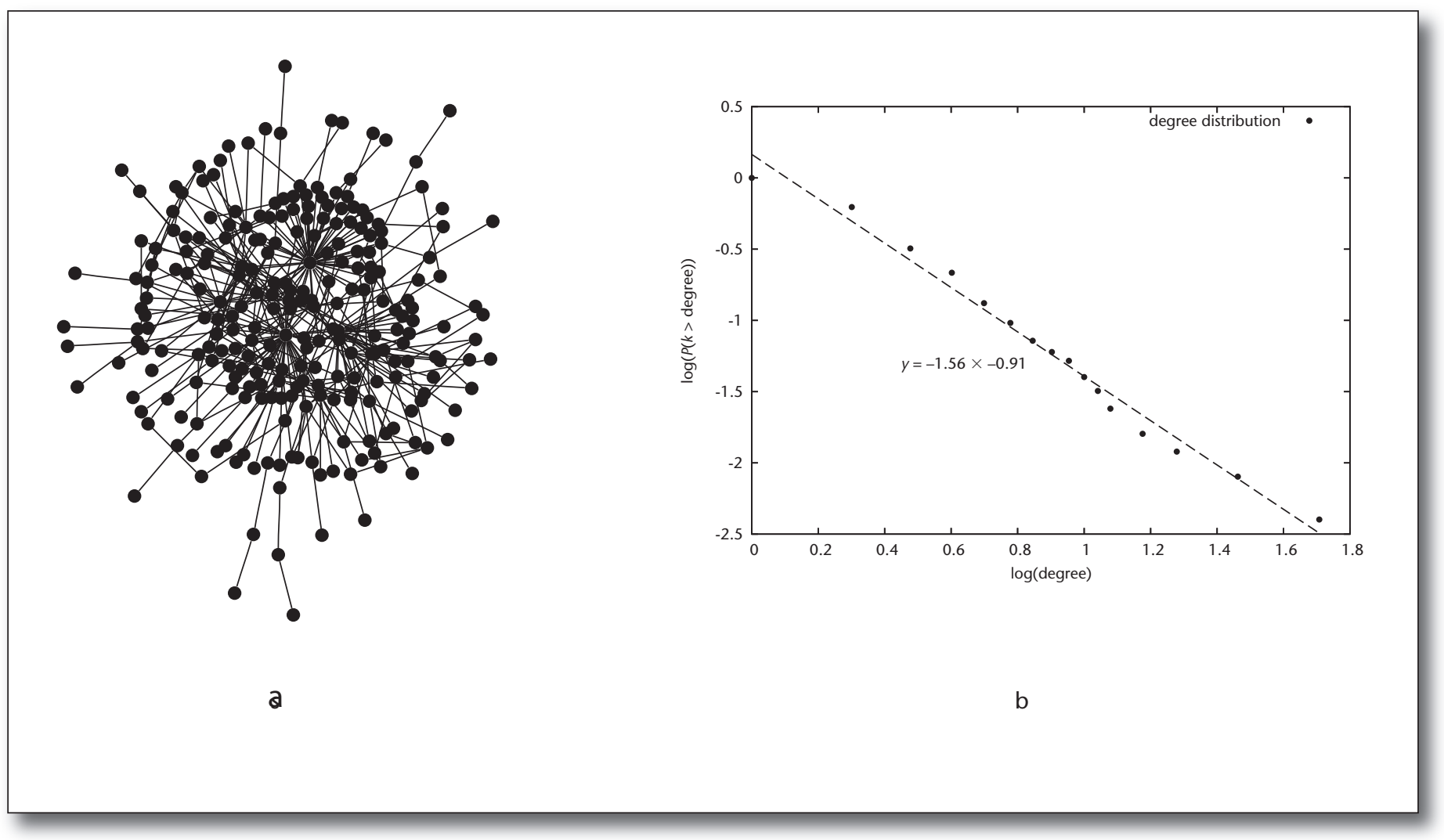

Figure 3. An Example of a Scale-Free Network Structure with 250 Nodes.

(a) A rendering of the network that clearly shows the hub-and-spoke structure. (b) A log-log plot of the cumulative degree distribution of the network shown in (a). Note that a linear curve in a log-log plot implies a power-law behavior of the underlying system.

Newman, M. 2003. The Structure and Function of Complex Networks. SIAM Review 45(2):167-256.

Watts, D. 1999. Small Worlds. Princeton, NJ: Princeton University Press.

Watts, D., and Strogatz, S. 1998. Collective Dynamics of "Small-World" Networks. Nature 393(6684): 440-442.

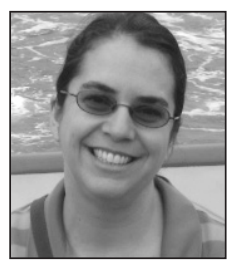

Marie desJardins (mariedj@cs.umbc. edu) is an associate professor in the Department of Computer Science and Electrical Engineering at the University of Maryland Baltimore County. Her research focuses on machine learning, planning, and multiagent systems, with an emphasis on interactive and integrated approaches to AI.

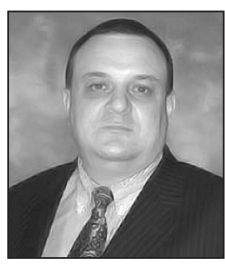

Dragomir R. Radev is an associate professor at the School of Information and the Department of Electrical Engineering and Computer Science at the University of Michigan. Radev received a Ph.D. in computer science from Columbia University in 1999. He is currently secretary of the Associa- tion for Computational Linguistics and program chair of the North American Computational Linguistics Olympiad and also coach of the US national linguistics teams. His areas of research include natural language processing, information retrieval, and network theory.

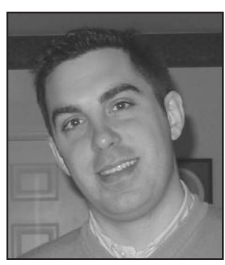

Matthew E. Gaston is a senior research scientist at Viz, a division of General Dynamics C4 Systems, where he leads research and development activities focused on creating intelligent user interfaces for information visualization and collaboration. He received his Ph.D. in computer science from the University of Maryland Baltimore County, where his research focused on networks and multiagent learning. He can be contacted at mgasto1@cs.umbc.edu. 